\title{
CONSTIPAÇÃO INTESTINAL NA CRIANÇA
}

\author{
CONSTIPATION IN CHILDREN
}

Rosa H. M. Bigélli', Maria I. M. Fernandes² \& Lívia C. Galvão²

${ }^{1}$ Médica Assistente. ${ }^{2}$ Docentes. Setor de Gastroenterologia Pediátrica. Departamento de Puericultura e Pediatria. Faculdade de Medicina de Ribeirão Preto - USP

CorRespondênCIA: Dra. Maria Inez Machado Fernandes. Departamento de Puericultura e Pediatria da Faculdade de Medicina de Ribeirão Preto - USP. Hospital das Clínicas de Ribeirão Preto. Av. Bandeirantes, 3900. CEP 14049-900

Fone: (016) 6022573 - Fax: (016) 602-2700 E-mail: mimferna@fmrp.usp.br

BIGÉLLI RHM; FERNANDES MIM \& GALVÃO LC. Constipação intestinal na criança. Medicina, Ribeirão Preto, 37: 65-75, jan./jun. 2004.

RESUMO: Objetivo - Apresentar uma revisão sobre a constipação intestinal na criança, analisando as principais características clínicas bem como sua abordagem diagnóstica e terapêutica.

Métodos - Revisão bibliográfica atualizada e experiência da Unidade de Gastroenterologia do Departamento de Pediatria- HCRP- USP.

Resultados - A constipação intestinal constitui um problema comum na prática pediátrica. Sua definição está baseada na eliminação de fezes ressecadas, com esforço e dor. É comum as crianças pequenas apresentarem choro durante as evacuações. Em algumas crianças, pode ser observada retenção fecal importante, com ou sem escape fecal. O diagnóstico, na maioria dos casos, pode ser feito através de dados de história clínica e exame físico cuidadoso dos pacientes. O tratamento deve ser direcionado à causa básica, necessitando, na maioria dos casos, mudanças dietéticas e comportamentais.

Conclusões - O diagnóstico da constipação intestinal deve ser precoce, pois interfere no sucesso terapêutico, que depende de uma adequada alimentação e do recondicionamento esfincteriano.

Unitermos: Constipação. Criança. Motilidade Gastrointestinal.

\section{1- INTRODUÇÃO}

A constipação intestinal constitui um problema comum na prática pediátrica. Estima-se que uma de cada dez crianças requeiram atenção médica para constipação, em alguma época da vida. ${ }^{(1)}$ Existem estimativas de que a população pediátrica seja acometida por constipação intestinal em taxas que variam entre 0,3 a $8,0 \%{ }^{(2,3)}$. Em Serviços de Gastroenterologia Pediátrica, 20,0 a 25,0\% das consultas são devidas à constipação intestinal ${ }^{(1,3,4,5)}$. No Brasil, estudos mais recentes de prevalência de constipação intestinal em escolares, em comunidade de baixa renda e em unidade básica de saúde, mostraram valores elevados, variando de $17,5 \%$ a $38,4 \%{ }^{(6,7,8,9)}$ (Tabela I).

\section{2- CONCEITO DE CONSTIPAÇÃO INTES- TINAL}

A constipação intestinal é, usualmente, definida em termos de mudanças na freqüência, tamanho, consistência ou facilidade de passagem das fezes ${ }^{(2,3)}$. Alguns estudos constataram que $85,0 \%$ das crianças entre um e quatro anos de idade tinham uma a duas evacuações por dia e 96,0\% apresentavam freqüência de evacuações, variando de três vezes ao dia até uma vez a cada dois dias ${ }^{(10,11)}$. Outros pesquisadores mostraram que nos primeiros três anos de vida, 97,0\% das crianças saudáveis tinham pelo menos uma evacuação a cada dois dias e, após o terceiro ano de vida, $95,0 \%$ das crianças tinham este padrão ${ }^{(12)}$. 
Tabela I- Estudos mais recentes de prevalência de constipação na população pediátrica brasileira

Autor/ cidade

Maffei et al. (1997) Botucatu, SP

Motta \& Silva (1998) Recife, PE

Del Ciampo (1999) Ribeirão Preto, SP
Local do estudo/ faixa etária

Escola 6 a 16 anos

Comunidade $<11$ anos

Unidade básica de saúde 1 a 10 anos
Prevalência de constipação

$28,8 \%-38,4 \%$

$17,5 \%$

$26,8 \%$
A constipação intestinal pode, assim, ser definida por uma frequiência fecal menor que três vezes por semana, mas é melhor definida como a ocorrência de evacuações dolorosas e eliminadas com esforço, comumente acompanhadas por choro, em crianças pequenas ou quando a criança apresenta retenção fecal, com ou sem escape, ainda que o número de evacuações seja maior ou igual a três vezes por semana. Em crianças com quatro anos ou menos, a razão entre meninas e meninos com constipação é de 1:1, enquanto, em pacientescom 5 anos ou mais, encontrou-se uma razão de $3-4: 1^{(3)}$.

\section{3- CLASSIFICAÇÃO DA CONSTIPAÇÃO IN- TESTINAL}

\section{1- Quanto ao tempo de duração}

A constipação pode ser classificada como aguda ou crônica. Não se encontra na literatura um limite de tempo para se diferenciar um quadro agudo de um crônico. É muito importante o reconhecimento precoce de um quadro agudo, visto que, na maioria dos casos, não se observa nenhuma anormalidade intestinal e o prognóstico é melhor que nos casos crônicos ${ }^{(13)}$. Um episódio agudo de constipação pode seguir-se a uma mudança de dieta ou ambiente, um período febril, um período de desidratação ou de repouso no leito ${ }^{(3)}$. Os quadros crônicos de constipação podem ser decorrentes de um manejo inadequado de uma constipação aguda. Em algumas crianças a constipação se desenvolve gradualmente como resultado de uma progressiva diminuição da freqüência das evacuações e um aumento progressivo da dificuldade na passagem de fezes excessivamente endurecidas. A motilidade colônica lenta intrínseca e o comportamento da criança em reter fezes, devido às evacuações dolorosas de fezes calibrosas, são fatores que contribuem para o estabelecimento da constipação crônica ${ }^{(2)}$.

\section{2- Quanto à etiologia}

A constipação pode ser classificada em orgânica e funcional ${ }^{(2)}$.

\subsection{1- Orgânicas}

São aquelas em que o fator etilológico é conhecido. Podem ser classificadas como a seguir:

- Causas neurogênicas - doença de Hirschsprung, pseudo-obstrução intestinal crônica, desordens do sistema nervoso central (SNC) como: meningomielocele, tumor, paralisia cerebral e hipotonia.

$\rightarrow$ Causas anais - fissuras, ânus anteriorizado, estenose e atresia anal.

จ Causas endócrinas e metabólicas - hipotireoidismo, acidose renal, diabete insípido e hipercalcemia.

๑ Uso de drogas - metilfenidato, fenitoína, imipramina, fenotiazida, antiácidos e medicamentos contendo codeína.

\subsection{2- Funcionais}

São aquelas em que o fator etiológico é desconhecido. Segundo descrições da literatura, correspondem a 95,0\% das constipações apresentadas pelas crianças $^{(14)}$.

\section{4- FISIOPATOLOGIA DA CONSTIPAÇÃO IN- TESTINAL CRÔNICA FUNCIONAL (CICF)}

\section{1- A fisiopatologia da CICF é desconhecida}

A habilidade para defecar depende de vários fatores, incluindo as ações simultâneas dos esfíncteres anais interno e externo, a tonicidade do reto e a condição da medula espinhal ${ }^{(14)}$ (Figura 1).

Várias alterações já foram descritas em crianças com CICF, tais como: pressão esfinctérica anor- 

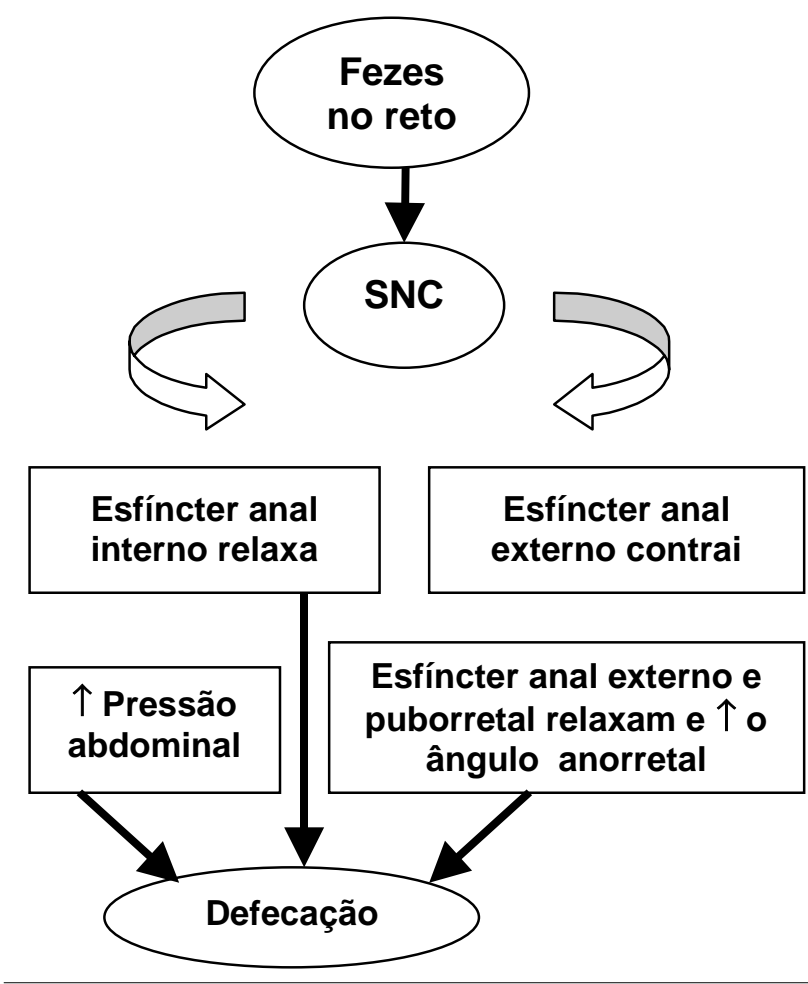

Figura 1- Mecanismo de defecação e continência.

malmente elevada ${ }^{(15)}$, sensibilidade retal reduzida ${ }^{(16,17)}$ e falta de relaxamento ou contração paradoxal do esfíncter anal externo ${ }^{(15,16,18)}$. Foram também citadas as anormalidades de plexo mientérico colônico como fator contributório na fisiopatologia da constipação intestinal crônica grave, embora, nesses casos, o exame histológico de rotina seja aparentemente nor$\operatorname{mal}^{(15,19)}$.

Alguns autores ${ }^{(20)}$ constataram redução na quantidade de peptídio intestinal vasoativo (VIP) e peptídeo histidinametionina (PHM) em músculo liso circular colônico de pacientes com CICF, enquanto outros ${ }^{(19)}$ verificaram aumento de serotonina na mucosa e aumento de ácido hidróxindolacético em mucosa e músculo circular de cólon sigmóide nesses pacientes. Já foi, também, descrita a diminuição na liberação de motilina em alguns casos de $\mathrm{CICF}^{(15)}$. Observou-se, também, em crianças com megarreto, dificuldade de propulsão de fezes do reto por peristalse e/ou atonia retal impedindo a defecação e sugeriu-se que, em crianças constipadas em que o escape fecal é frequiente, o problema básico possa ser uma anormalidade do esfíncter anal interno, que é mais fraco e menos responsivo à distensão retal, quando comparado com o das crianças não constipadas ${ }^{(21)}$.
5- QUADRO CLÍNICO DA CICF (Tabelas II e III)

As dificuldades de defecação afetam crianças em todas as faixas etárias, sendo mais freqüentes nas crianças entre um e cinco anos de idade. Na literatura, encontrou-se que $15,0 \%$ tornam-se constipadas entre zero e um ano de idade, 70,0\% entre um e cinco anos e $15,0 \%$ com mais de cinco anos de idade ${ }^{(22)}$.

A forma de apresentação clínica da constipação varia com o período de vida em que a criança se encontra. Dentro do período compreendido entre os dois primeiros anos de vida e todo o período pré-escolar a frequiência de constipação entre meninos e meninas é de 1:1. A constipação crônica é decorrente do manejo inadequado de um problema agudo. À passagem de fezes endurecidas, ressecadas, em cíbalos, há dor e a criança começa a reter fezes na tentativa de evitar desconforto. O reto acomoda o conteúdo fecal e a urgência para defecar passa. $O$ ciclo vai se repetindo, de modo que se vai, sucessivamente, eliminando fezes mais calibrosas, endurecidas e dolorosas, o que é manifestado clinicamente, como choro e grito às evacuações, contração dos músculos glúteos e anais, extensão do corpo, recusa de se sentar no vaso sanitário e várias outras manobras com a finalidade de evitar a evacuação ${ }^{(2)}$.

Tabela II - Sintomas associados à constipação intestinal crônica funcional

Sintomas

Porcentagem

Dor abdominal

$10-50 \%$

Falta de apetite

$26 \%$

Queixas urinárias

$15 \%$

Problemas psicológicos

$20 \%$

Tabela III- Sinais associados à constipação intestinal crônica funcional

Sintomas

Porcentagem

Peso < Percentil 5

$0-10 \%$

Escape fecal

4- $8 \%$

Distensão abdominal

$20 \%$

Massa abdominal

30- $50 \%$

Impactação fecal

40- $80 \%$

Dilatação retal

$18 \%$

Fissura anal

$26 \%$

Prolapso retal

$5 \%$ 
As crianças em idade escolar, comumente, são levadas para consulta médica devido a escape fecal. O escape é, usualmente, decorrente de uma constipação de longa duração ${ }^{(2)}$. O escape fecal é definido como perda involuntária de fezes nas roupas, em crianças acima dos quatro anos de idade, conseqüente à impactação fecal no reto. Considera-se a idade de quatro anos, pois se espera que até então a criança já esteja apta para controlar o esfíncter anal ${ }^{(14)}$.

A constipação é acompanhada de escape fecal em $4,0 \%$ a $8,0 \%$ da população pediátrica ${ }^{(23)}$. Além do escape fecal, pode-se notar, nas crianças em idade escolar, a presença de evacuações incompletas e evacuações periódicas de fezes muito calibrosas e a recusa a se sentar no vaso sanitário. Podem ocorrer episódios de dor abdominal, precedendo as evacuações, vários dias antes de evacuações de fezes dolorosas, ou diariamente. Muitos pacientes constipados têm dor abdominal recorrente. A anorexia só é presente em constipação crônica mais grave ${ }^{(24)}$. Evidenciou-se a presença de dor abdominal em 10,0\% a 50,0\% das crianças, falta de apetite em 26,0\% dos casos, queixas urinárias (infecções do tracto urinário e enurese) em $15,0 \%$ dos casos e problemas psicológicos em 20,0\% dos casos (que são freqüientemente secundários à obstipação) ${ }^{(22,25)}$.

Ao exame físico desses pacientes, podemos detectar: peso abaixo do percentil 5 em até $10,0 \%$ dos casos, sintomas de retenção fecal crônica, distensão abdominal em 20,0\%; massa abdominal em 30,0\% a $50,0 \%$; e impactação fecal em $40,0 \%$ a $80,0 \%$ dos casos. A massa abdominal é mais comumente palpável na região suprapúbica e na linha média, podendo ocupar completamente o quadrante abdominal inferior direito e esquerdo ${ }^{(2)}$.

O exame anorretal é muito importante nos quadros de CICF permitindo excluir estenoses anais, ânus imperfurado e anomalias espinhais, maiores como agenesia sacral ${ }^{(13)}$. A dilatação anal está presente em $18,0 \%$ das crianças constipadas, a fissura em $26,0 \%$ e o prolapso retal em 5,0\% dos casos. Em 3/4 das crianças há dilatação anal, impactação fecal ou sinais perianais (fissuras, vermelhidão e arroxeamento) ${ }^{(26)}$.
Ao exame digital do reto, na CICF, normalmente se constata uma ampola retal cheia de fezes de consistência endurecida ou, mais comumente, a superfície externa da impactação tem consistência de "argila" e o centro têm consistência de "rocha". Não se percebe, porém, impactação fecal em crianças que em período recente ao exame físico, tiveram evacuações, contendo fezes calibrosas ${ }^{(2)}$.

\section{6- INVESTIGAÇÃO DIAGNÓSTICA DA CICF}

Na verdade, o diagnóstico, na CICF, é obtido, na maioria dos casos, através de dados de história clínica e exame físico cuidadoso. Os exames laboratoriais são utilizados quando se suspeita de uma causa orgânica de constipação, principalmente, da doença de Hirschsprung (Tabela IV) ou quando se quer estudar melhor os mecanismos fisiopatológicos envolvidos em um quadro de CICF.

Dentre os exames laboratoriais mais comumente utilizados, podem-se destacar: exames bioquímicos, estudos radiológicos (raio x simples abdominal, enema opaco), manometria anorretal e biópsia retal.

Tabela IV- Diagnóstico diferencial entre doença de Hirschsprung (forma clássica) e constipação intestinal crônica funcional

\begin{tabular}{|l|c|c|}
\hline & $\begin{array}{c}\text { Constipação } \\
\text { intestinal } \\
\text { crônica funcional }\end{array}$ & $\begin{array}{c}\text { Doença de } \\
\text { Hirschsprung } \\
\text { (forma clássica) }\end{array}$ \\
\hline Retenção fecal desde o nascimento & rara & sempre \\
\hline Escape fecal & presente & ausente \\
\hline Estado nutricional comprometido & incomum & comum \\
\hline Massa fecal abdominal & comum & incomum \\
\hline Fezes na ampola retal & comum & incomum \\
\hline Enema opaco & & \\
\hline - Segmento espástico & ausente & presente \\
\hline Manometria anorretal & & \\
\hline - Tônus do esfíncter anal & & aumentado \\
\hline - Reflexo retoesfincteriano & presentes & ausentes \\
\hline Biópsia retal & presente & ausente \\
\hline - Células ganglionares & & \\
\hline - Atividade da acetilcolinesterase & & \\
\hline
\end{tabular}




\section{1- Exames bioquímicos}

São indicados quando se suspeita de deficiências ou excessos de hormônios tireoidianos ou adrenais e distúrbios eletrolíticos.

\section{2- Exames radiológicos}

Dentre os exames radiológicos, destacam-se: o raio x simples abdominal, indicado quando se suspeita de constipação intestinal crônica, quando a história clínica não elucida o diagnóstico e, ao toque retal, não se palpam fezes na ampola retal. Ele avalia a presença ou ausência de retenção fecal e sua extensão, caso ela esteja presente ${ }^{(27)}$. Avalia, também, se a parte mais baixa de espinha é normal em criança com escape fecal, em que está ausente a massa fecal no abdômen e reto.

O enema opaco é indicado, quando há forte suspeita de doença de Hirschsprung ou outras desordens neuronais, ou seja, em crianças com história de atraso na eliminação de mecônio, em que a constipação se iniciou em idade muito precoce (principalmente nas < 6 meses), com distensão abdominal importante, com toque retal evidenciando uma zona de estreitamento (chamado sinal do "dedo de luva") e, à retirada do dedo, eliminação explosiva de fezes e, finalmente, nas crianças que sendo conduzidas como tendo CICF, não têm boa resposta à terapêutica. É desnecessário nos quadros não complicados de constipação, mas é útil no estabelecimento da doença de Hirschsprung, em que a zona de transição entre o segmento intestinal agangliônico e o gangliônico pode ser visualizada, e de outras desordens neuronais em que uma dilatação intestinal extensa possa ser vista ${ }^{(2)}$.

\section{3- Manometria anorretal}

Desde 1935, com os clássicos experimentos de Denny Brown \& Robertson, o procedimento manométrico tem provado ser o mais apropriado instrumento no estudo da motilidade anorretal ${ }^{(28)}$. Atualmente, a manometria anorretal é um exame bastante utilizado no paciente pediátrico e bem reconhecido por ajudar no estabelecimento do diagnóstico diferencial da constipação intestinal, incontinência fecal e na avaliação pré e pós operatória de anomalias retais congênitas, adquiridas ou traumáticas ${ }^{(29,30,31)}$. Os achados manométricos mais comumente verificados nas crianças com CICF e escape fecal são: hipertonia anal, hipotonia anal, falta de relaxamento do esfíncter anal externo e assoalho pélvico durante a tentativa de defecação, habilidade diminuída do esfíncter anal interno, para relaxar completamente durante a distensão retal, aumento da complacência retal e do limiar de sensibilidade retal consciente, além de diminuição da contratilidade retal ${ }^{(16,17,21,32 / 37)}$. A manometria anorretal, na investigação da constipação intestinal crônica, tem, basicamente, as mesmas indicações do enema opaco, tendo importância destacada nos casos de doença de Hirchsprung ultracurta, em que o enema opaco é normal, embora, na manometria anorretal não se observe o reflexo reto esfincteriano.

\section{4- Biópsia retal}

Utilizada, basicamente, quando na avaliação clínica e ou laboratorial da criança constipada (estudos radiológicos e manométricos dentre outros), há forte suspeita de que a constipação seja de causa orgânica, sendo as mais importantes a doença de Hirschsprung e as displasias neuronais. É considerado o exame-padrão no diagnóstico da doença de Hirschsprung. A biópsia retal pode ser feita por sucção superficial ou biópsia de camada completa. No material de biópsia de reto por sucção, pode ser utilizada a coloração pelo método tradicional hematoxilina e eosina, bem como o método histoquímico para a acetilcolinesterase na doença de Hirschsprung, em que se encontra aumento significativo do número e do tamanho das fibras nervosas colinérgicas na lâmina própria e muscular da mucosa. O teste foi confirmado como um dos mais confiáveis métodos existentes para confirmar ou afastar a suspeita clínicorradiológica da doença de Hirschsprung ${ }^{(38)}$. Pela biópsia de camada completa, é possível avaliar as anormalidades presentes no plexo submucoso e mientérico, como: aganglionose, hipoganglionose e hiperganglionose ${ }^{(39)}$.

\section{7- TRATAMENTO DA CONSTIPAÇÃO IN- TESTINAL}

\section{1- Aguda}

Nos quadros de constipação aguda, a criança tem boa resposta quando se faz a correção de fatores precipitantes e o tratamento sintomático. Deve-se aumentar o aporte de fibras na dieta. Quando só a dieta não é suficiente para o controle do quadro, deve-se instituir a utilização de laxativos por períodos curtos (3-4 dias). Raramente, necessita-se do uso de enemas ou supositórios ${ }^{(13)}$. 


\section{2- Crônica}

\subsection{2- Orgânica}

Deve ser direcionada a causa básica. Sendo assim, pode-se citar como exemplos: reposição de hormônios tireoidianos, no hipotireoidismo; retirada do segmento agangliônico na doença de Hirschsprung clássica.

\section{Funcional}

O manejo da constipação crônica funcional, na infância, tem um número de problemas e desafios para o pediatra ${ }^{(40)}$. Antes de iniciar o tratamento, é importante a elaboração de um plano organizado e bem fundamentado. O objetivo de cada conduta a ser instituída deve ser explicado aos pais ou responsáveis pela criança e, se possível, inclusive, à própria criança, em uma linguagem apropriada ao entendimento de todos, a fim de que todo o plano possa ser realizado integralmente e de forma adequada. Existem, basicamente, quatro tipos de tratamento, atualmente propostos no manejo dos quadros funcionais de constipação: tratamento clínico (convencional), tratamento psicológico, tratamento através do biofeedback e tratamento cirúrgico.

\subsection{2.a- Tratamento clínico (convencional)}

Pode ser dividido em quatro fases: educação, desimpactação, prevenção de reacumulação de fezes e recondicionamento para hábitos intestinais normais $^{(2,14,40,41)}$.

\section{- Educação}

Explicar aos pais o motivo da constipação e do escape fecal apresentados pelas crianças, enfatizando que o escape é involuntário e, usualmente, ocorre sem o conhecimento do paciente. O escape fecal não é um distúrbio psicológico ou comportamental das crianças, nem é culpa dos pais.

\section{- Desimpactação}

Pode ser feita através de lavagens intestinais, utilizando-se soro fisiológico com glicerina a 10,0\%, soro fisiológico e óleo de cozinha na proporção de 2: $1^{(42)}$ ou com enemas fosfato-hipertônicos, na quantidade de $10 \mathrm{ml} / \mathrm{Kg}$. No caso de enema, utiliza-se, no máximo, $100-120 \mathrm{ml}$ da solução. Geralmente, uma ou duas lavagens intestinais são suficientes para o clareamento intestinal. Os enemas de fosfato-hipertônicos podem levar à criança à desidratação, além de aparecerem distúrbios eletrolíticos como: hipernatremia, hiperfosfatemia, hipocalcemia e hipocalemia. Devido a isso, pre- fere-se utilizá-los em crianças acima de dois anos de idade. A desimpactação inicial também pode ser feita através da ingestão oral de grandes doses de óleo mineral (15-30ml por ano de idade, por dia, com um máximo de $240 \mathrm{ml}$ ) ou através de outras soluções balanceadas, dadas por boca ou por sonda nasogástrica, em crianças que temem e recusam a utilização da via anorretal para a desimpactação ${ }^{(43)}$.

\section{- Prevenção da reacumulação de fezes}

É feita através da utilização de fibras alimentares, ingestão hídrica e uso de laxativos. Existem dois tipos de fibras: as solúveis e as insolúveis. Ambas são importantes no tratamento da CICF. Os alimentos que encerram, proporcionalmente, maiores quantidades de fibras solúveis são: os legumes, aveia, maçãs e frutas cítricas, enquanto as fibras insolúveis são encontradas em maiores proporções, no farelo de trigo e nos cereais. Uma dieta equilibrada, composta de cereais, frutas e folhas verdes guarda uma relação fibra-insolúvel: solúvel de 3:1(44). O Comitê de Nutrição da Academia Americana de Pediatria recomenda que a quantidade de fibra alimentar seja da ordem de $0,5 \mathrm{~g} / \mathrm{Kg} / \mathrm{dia}$ ou seja, aproximadamente $10 \mathrm{~g} / 1000 \mathrm{Kcal}$, atingindo um valor-limite na adolescência, de 30g/dia. A Fundação Americana de Saúde (American Health Foundation) preconiza que, a partir do término do período de lactência até atingir a idade adulta, a ingestão diária de fibra deva ser a idade em anos, acrescida de $5 \mathrm{~g}$, atingindo o máximo de $25 \mathrm{~g}$, no período pubertário. Nos quadros de CICF, observa-se, pelo diário alimentar, que a maioria das crianças apresenta uma alimentação com baixa quantidade de fibras. O aleitamento natural é fator de proteção contra a constipação no $1^{\underline{0}}$ semestre de vida. Deve-se, portanto, em lactentes desmamados, prevenir a constipação, orientando a introdução precoce de fibras alimentares, como cereais e frutas. Desde que não esteja mais recebendo leite humano, não existe limite inferior de idade para a introdução de fibra alimentar na dieta de lactentes, a fim de prevenir a constipação intestinal, já que esta freqüentemente se inicia com o desmame ${ }^{(45,46,47)}$. As fibras utilizadas para o tratamento da constipação podem ser as encontradas nos próprios alimentos ou através de medicamentos. Sempre damos preferência às não medicamentosas, já que são mais saborosas e, também, mais fáceis de serem usadas na alimentação da criança. A vantagem do uso de fibras medicamentosas está no fato de que, em uma pequena quantidade de medicamento, consegue-se concentrar uma grande quantidade de fibras. Mas, apesar do sabor ser bem aceito 
pelas crianças, no início da terapêutica, no decorrer da mesma, gera intolerância com grande freqüência. A quantidade de fibras fornecidas à criança, durante $\mathrm{o}$ tratamento é geralmente empírica, pois é muito difícil determinar com exatidão, quanto, em gramas, a criança está ingerindo, principalmente devido ao fato de as crianças constipadas terem uma alimentação muito irregular e inadequada. Procura-se, assim, no momento em que se institui a alimentação com fibras, como medida terapêutica, corrigir a alimentação da criança tornando-a adequada qualitativa e quantitativamente e, também, acertando os horários das refeições. Assim, espera-se, pelo menos, estar fornecendo uma quantidade de fibras que esteja atendendo às necessidades do paciente.

Ingestão Hídrica - o fornecimento de líqüidos é muito importante na determinação da consistência fecal. Assim, deve-se fazer uma rigorosa recomendação de ingestão hídrica, em crianças constipadas, de cerca de 1 a 21 de líqüido por dia.

Laxativos - devem ser iniciados já no dia da primeira consulta da criança. Eles são utilizados, sempre levando em consideração a idade, o peso e a gravidade da constipação. Os laxativos são preferencialmente, usados em crianças acima de seis meses de idade. Abaixo dos seis meses, a modificação da dieta costuma regularizar a função intestinal das crianças com constipação funcional e, é arriscado o uso de laxantes devido aos efeitos colaterais dos mesmos. Atualmente, acredita-se que a escolha da medicação seja menos importante que a aderência dos pais e crianças à terapêutica instituída. Há somente uma dose inicial para cada criança, que deve ser ajustada para induzir uma a duas evacuações diárias com eliminação de fezes, que garanta o esvaziamento diário e completo do intestino grosso e previna escape fecal e dor abdominal. Os laxativos mais comumente utilizados são: o óleo mineral o leite de magnésia e a lactulona, na dose de $1-2 \mathrm{ml} / \mathrm{Kg} / \mathrm{dia}$, uma a duas vezes ao dia.

\section{- Recondicionamento da criança para há- bito intestinal normal}

Quando a criança já adquiriu controle esfincteriano, procura-se incentivá-la a sentar no vaso sanitário, a fim de aproveitar a presença do reflexo gastrocólico, que facilita as evacuações. Sendo assim, orienta-se que a criança sente no vaso sanitário uma a três vezes ao dia, após o café da manhã e grandes refeições (almoço e jantar), por um período de 5 a 10 minutos para tentar defecar. O recondicionamento é programado após ter sido feita a desimpactação e ter sido iniciado o uso de óleo mineral pois assim, a evacuação torna-se mais fácil e indolor.

No acompanhamento da criança constipada, é essencial que se esteja sempre verificando a adequação da criança às medidas terapêuticas instituídas (orientação alimentar, condicionamento esfincteriano, uso de laxativos) e a resposta da mesma ao tratamento instituído; para isto, pode-se fazer uso de diários alimentares e diários de frequiência e características das evacuações e do escape, assim os pais e as próprias crianças tornam-se mais envolvidos e mais estimulados na resolução da constipação. Além dos diários, são importantes os retornos médicos que, no início, devem ser mais próximos e, depois, podem ser mais espaçados, a medida que os pais e crianças já compreenderam a dinâmica da terapêutica. Nas consultas médicas, além da conversa com os pais e os pacientes, o médico dispõe do exame abdominal e retal, para se assegurar de que a constipação está sendo adequadamente tratada, fazendo-se os ajustes necessários nas doses de laxativos a fim de garantir hábito intestinal regular. Após se conseguir um hábito intestinal regular, a dose do laxativo é gradualmente reduzida, de modo a se manter um hábito intestinal diário, sem escape ${ }^{(2)}$.

\subsection{2.b- Tratamento Psicológico}

Os distúrbios psicológicos, apresentados pelas crianças constipadas são, na maioria das vezes, secundários ao quadro clínico da própria constipação intestinal. Destaca-se, como sinal mais importante no desencadeamento desses distúrbios, o escape fecal. Sendo assim, a resposta satisfatória à terapêutica da constipação, com a eliminação dos sinais e sintomas, bem como os esclarecimentos necessários e apoio dados pelo próprio médico que cuida da criança são, geralmente, suficientes para resolver os distúrbios psicológicos. Opta-se, porém, para a intervenção de psicólogos e psiquiatras quando não se encontra adesão ao tratamento e/ou o paciente apresenta distúrbios comportamentais que não melhoram com a atuação exclusiva do médico que está cuidando da criança ${ }^{(48)}$.

\subsection{2.c- Tratamento Biofeedback}

Tal tratamento foi difundido a partir de 1986 quando, através de estudos manométricos, Robinson 
e Gibbons apud KEREN et al. (1988) (36) demonstraram a contração do esfíncter anal externo, durante a tentativa de defecação em crianças com constipação crônica e escape fecal. O biofeedback visa treinar o paciente a relaxar o esfíncter anal externo durante o ato do esforço para a evacuação. A técnica envolve a colocação de um tubo de metal, de plástico ou de borracha, atado a balões de látex, dentro do canal anal e reto, que monitoriza a contração paradoxal do esfíncter anal externo, durante ou a distensão do balão retal ou a tentativa voluntária de esforço para a evacuação. Estudos do uso do biofeedback, em crianças com constipação intestinal e contração paradoxal do esfíncter anal externo, são concordantes em evidenciar que essa forma de tratamento é superior ao tratamento convencional, usado, isoladamente, na recuperação das crianças com constipação, quando elas são acompanhadas a curto prazo, ou seja, em torno de um ano $^{(49 / 53)}$. Já, na avaliação a longo prazo, outros estudos são necessários. Um estudo que seguiu crianças constipadas com contração paradoxal do esfíncter anal externo, por um período médio de quatro a cinco anos, não evidenciou maior taxa de recuperação em crianças tratadas apenas convencionalmente $(86,0 \%)$ que a das crianças tratadas com a associação entre o tratamento convencional e o biofeedback $(87,0 \%)^{(54)}$. No entanto, outros autores, ${ }^{(55)}$ acompanhando crianças com constipação funcional e contração paradoxal do esfíncter anal externo, que não responderam à terapêutica convencional por um período médio de 23 meses, observaram melhora da constipação em 57,0\% dos casos, mostrando que o biofeedback tornou-se uma possibilidade terapêutica nas crianças com contração paradoxal do esfíncter anal externo, que não são responsivas às medidas terapêuticas tradicionais.

\subsection{2.d- Tratamento cirúrgico da CICF}

Há alguns anos, a utilização do tratamento cirúrgico, nos quadros de constipação, era basicamente destinada aos casos de constipação decorrentes da doença de Hirschsprung e malformações anorretais ${ }^{(56,57)}$. Atualmente, a cirurgia tornou-se uma das modalidades terapêuticas para pacientes apresentando constipação crônica de causas não orgânicas. A cirurgia não é o tratamento de escolha para todos os tipos de constipação porém, pode oferecer uma opção terapêutica às pessoas que, desesperadas, buscam uma solução para sua constipação, que foi rotulada como "intratável" devido ao fato de apresentar trânsito lento ou a chamada obstrução de saída ${ }^{(58)}$. Nas crianças, o tratamento cirúrgico restringe-se, principalmente, aos casos de megacólon e megarreto, quando se emprega a colectomia parcial ${ }^{(59,60)}$ e a miomectomia do esfíncter anal interno em crianças portadoras de hipertonia do mesmo $^{(61)}$, com resultados favoráveis.

\subsection{2.e- Resposta da Criança com CICF ao Tra- tamento}

Os critérios utilizados para definir recuperação ou não recuperação de crianças com constipação crônica funcional são variáveis de autor para autor. Enquanto alguns autores definem, como critério de recuperação, a freqüência de evacuações maior ou igual a três evacuações por semana sem escape fecal, em crianças não usando medicamentos ou qualquer outro tratamento por pelo menos um mês, outros consideram curadas as crianças com, pelo menos, cinco evacuações por semana sem escape fecal e com uso ocasional de laxativos (menos que uma vez por semana) e melhoradas, as crianças com, pelo menos, três evacuações por semana e que apresentem escape fecal menos de uma vez por semana ${ }^{(2,62)}$. Em um estudo de seguimento a longo prazo (6,9+/-2,7 anos), de 90 crianças constipadas, que foram, inicialmente, avaliadas e tratadas, quando menores ou iguais a quatro anos de idade, observou-se que, com $63,0 \%$ houve recuperação, sendo que a taxa de recuperação em crianças menores ou iguais a dois anos de idade é maior que entre dois e quatro anos de idade, mostrando que crianças menores que dois anos de idade respondem melhor à terapêutica. Sendo assim, conclui-se que o tratamento da constipação deva ser feito o mais precoce possível, a fim de prevenir o desenvolvimento de um quadro de constipação grave e/ ou escape fecal. Já na avaliação de 52 crianças com CICF, entre quatro e doze anos de idade verificou-se recuperação em 50,0\%, alguma melhora em $20,0 \%$ e $25,0 \%$ permaneciam com constipação e escape fecal, após 3,1+/-0,4 anos do início do tratamento convencional ${ }^{(21)}$. A recuperação ou não recuperação da CICF, com ou sem escape fecal, é dependente da gravidade da obstipação manifestada, bem como das anormalidades evidenciadas na manometria anorretal. Dentre os fatores mais comumente estudados, que se acredita estarem envolvidos na resposta terapêutica à constipação, destacam-se: problemas psicológicos, nível sócio-econômico e cultural, complacência ao tratamento, gravidade da constipação, anormalidades no funcionamento dos esfíncteres anais interno e externo, alterações na contratilidade e sensibilidade retais ${ }^{(14,16,17,21,25,62)}$. 
BIGÉLLI RHM; FERNANDES MIM \& GALVÃO LC. Constipation in children. Medicina, Ribeirão Preto, 37: 65-75, jan/june. 2004.

Abstract: Objective: To present an update review of intestinal constipation in children, analysing the principal clinic characteristics and its diagnostic and therapeutic approachs.

Methods: Recent articles on the topic were reviewed, supplemented by the clinical experience of the Gastroenterology Unit, of the Pediatric Departament- HCRP- USP

Results: The intestinal constipation is a commun problem in the pediatric clinics. Its definition is based on the elimination of painful, difficulted and dry feces. Infants usually present crying during the evacuations. In some children it can be observed important facael retention with or without soiling. The diagnosis is based on a deitailed clinical history and physical examination of the children. The diagnosis in the most of the cases may be done with a carefully clinical history and phisical examination. The treatment must be done according to the basal cause and in the most of the cases dietetic and comportamental changes are necessary.

Conclusões: The diagnosis of the intestinal constipation must be done earlier because it is important for the therapeutic sucess, that depends on a adequate diet and a behavioral program to regulate bowel habits.

Uniterms: Constipation. Children. Gastrointestinal Motility.

\section{REFERÊNCIAS BIBLIOGRÁFICAS}

1 - DI LORENZO C; FLORES AF; REDDY SN \& HYMAN PE. Use of colonic manometry to differentiate causes of intractable constipation in children. J Pediatr 120: 690-695, 1992.

2 - LOENING-BAUCKE VA. Chronic constipation in children. Gastroenterology 105: 1557-1564, 1993a.

3 - LOENING-BAUCKE VA. Constipation in early childhood: patient characteristics, treatment and longterm follow up. Gut 34: 1400-1404, 1993b.

4 - MURRAY RD; LI BUK; MACCLUNG HJ; HEITLINGER L \& REHM D. Cisapride for intractable constipation in children: observations from an open trial. J Pediatr Gastroenterol Nutr 11: 503-508, 1990.

5 - NURKO S; GARCIA-ARANDA JA; GUERRERO VY \& WORONA LB. Treatment of intractable constipation in children: experience with cisapride. J. Pediatr Gastroenterol Nutr 22: 38-44, 1996.

6 - MAFFEI HVL; MOREIRA FL; OLIVEIRA Jr WM \& SANINI, V. Prevalência de constipação em escolares do ciclo básico. J Pediatr (Rio de Janeiro) 73: 340-344, 1997.

7 - MOTTA MEFA \& SILVA GAP. Constipação intestinal crônica funcional na infância: diagnóstico e prevalência em uma comunidade de baixa renda. J Pediatr (Rio de Janeiro) 74: 451-454, 1998

8 - MORAIS MB \& MAFFEI HVL. Constipação intestinal. J Pediatr (Rio de Janeiro.) 76: 147-156, 2000. Supl.2

9 - DEL CIAMPO IRL. Constipação intestinal crônica na infância: prevalência, diagnóstico e valorização de seus sinais e sintomas e unidade básica de saúde. Dissertação de Mestrado, Faculdade de Medicina de Ribeirão Preto da USP, Ribeirão Preto. p.1-116, 1999.
10 - WEAVER LT \& STEINER H. Bowel habit of young children. Arch Dis Child 59: 649-652, 1984.

11 - WEAVER LT. Bowel habit from birth to old age. J Pediatr Gastroenterol Nutr 7: 637-640, 1988.

12 - FONTANA M; BIANCHI C; CATALDO F; CONTI NIBALI S; CUCCHIARA S; GOBIO CASALI L; IACONO G; SANFILIPPO $M$ \& TORRE $G$. Bowel frequency in healthy children. Acta Paediatr Scand 78: 682-684, 1989.

13 - CLAYDEN GS. Constipation and soiling in childhood. Br Med J 1: 515-517, 1976

14 - LOENING-BAUCKE VA. Encoprese and soiling. Pediatr Clin North Am 43: 279-298, 1996.

15 - DAHL J; LINDQUIST BL; TYSK C; LEISSNER P; PHILIPSON L \& JÄRNEROT G. Behavioral medicine treatment in chronic constipation with paradoxical anal sphincter contraction. Dis Colon Rectum 34: 769-776, 1991.

16 - LOENING-BAUCKE VA \& CRUIKSHANK BM. Abnormal defecation dynamics in chronically constipated children with encopresis. J Pediatr 108: 562-566, 1986.

17 - DE MEDICI A; BADIALI D; CORAZZIARI E; BAUSANO G \& ANZINI, F. Rectal sensitivity in chronic constipation. Dig Dis Sci 34: 747-753, 1989.

18 - SHOULER P \& KEIGHLEY MRB. Changes in colorectal function in severe idiopathic chronic constipation. Gastroenterology 90: 414-420, 1986.

19 - LINCOLN J; CROWE R; KAMM MA; BURNSTOCK G \& LENNARD-JONES JE. Serotonin and 5-hydroxyindoleaceatic acid are increased in the sigmoid colon in severe idiopathic constipation. Gastroenterology 98: 1219-1225, 1990.

20 - KOCH TR; AIDAN CARNEY J; GO L \& GO VLW. Idiopathic chronic constipation is associated with decreases colonic vaso active intestinal peptide. Gastroenterology 94: 300310, 1988. 
21 - LOENING-BAUCKE VA. Abnormal rectoanal function in children recovered from chronic constipation and encopresis. Gastroenterology 87: 1229-1304, 1984.

22 - HATCH TF. Encopresis and constipation in children. Pediatr Clin North Am 35: 257-280, 1988.

23 - COX DJ; SUTPHEN J; BOROWITZ S; DICKENS MN; SINGLES $\mathrm{J} \&$ WHITEHEAD WE. Simple eletromiographic biofeedback treatment for chronic pediatric constipation/ encopresis: preliminary report. Biofeedback Self Reg 19: 41-50, 1994.

24 - MOTTA MEFA \& SILVA GAP. Sinais e sintomas associados à constipação crônica. J Pediatr (Rio de Janeiro) 76: 227-232, 2000

25 - LOENING-BAUCKE VA. Factors determining outcome in children with chronic constipation and facael soiling. Gut 30: 999-1006, 1989.

26 - AGNARSSON V; WARDE C; MCCARTHY G \& EVANS N. Perianal appearance associated with constipation. Arch Dis Child 65: 1231-1234, 1990.

27 - ROCKNEY RM; McQUADE WH \& DAYS AL. The plain abdominal roentgenogram in management of encopresis. Arch Pediatr Adolesc Med 149: 623-627, 1995.

28 - MEUNIER PD \& GALLAVARDIN D. Anorectal manometry: the state of the art. Dig Dis Sci 11: 252-264, 1993.

29 - MARTINS JL \& ESTEVES E. Computerized anorectal manometry in the pediatric patient- inicial experience. Rev Hosp São Paulo Esc Paul Med 6: 83-86, 1995.

30 - MARTINS JL \& PINUS J. Anorectal manometry in the child assessment of 1153 examinations performed from 1987 to 1995. Rev Hosp São Paulo Esc Paul Med 6: 80-82, 1995.

31 - KAUL A \& RUDOLPH, CD. Gastrointestinal manometry studies in children. J Clin Gastroenterol 27: 187-191, 1998.

32 - MEUNIER PD; MARECHAL JM \& DE BEAUJEU MJ. Rectoanal pressures and rectal sensitivity studies in chronic childhood constipation. Gastroenterology 77: 330-336, 1979.

33 - MEUNIER PD; MOLLARD P \& MARECHAL JM Physiopatology of megarectum: the association of megarectum with encopresis. Gut 17: 224-227, 1976.

34 - LOENING-BAUCKE VA \& YOUNOSZAI MK. Abnormal anal sphincter response in chronically constipated children. $\mathbf{J}$ Pediatr 100: 213-218, 1982.

35 - WALD A; CHANDRA R; CHIPONIS D \& GABEL, S. Anorectal function and continence mechanisms in childhood encopresis. J Gastroenterol Nutr 5: 346-351, 1986

36 - KEREN S; WAGNER Y; HELDENBERG D \& GOLAN, M. Studies of manometric abnormalities of the rectoanal region during defecation in constipated and soiling children: modification through biofeedback therapy. Am J Gastroenterol 83: 827-831, 1988.

37 - DA COSTA PINTO EAL; BUSTORFF SILVA, JM \& FUKUSHIMA, E. Papel da manometria anorretal no diagnóstico diferencial da constipação em crianças. J Pediatr (Rio de Janeiro) 76: 227-232, 2000.

38 - GUGELMIN ES; TORRES LFB \& SABAGGA CC. Aplicação da biópsia de reto por sucção no diagnóstico da doença de Hirschsprung. J Pediatr (Rio de Janeiro.) 66: 171-174, 1990.
39 - SWENSON O; FISHER JH \& MacMAHON, HE. Rectal biopsy as an aid in the diagnosis of Hirschsprung's disease. $\mathbf{N}$ Engl J Med 253: 632-635, 1955.

40 - CLAYDEN G S. Management of chronic constipation. Arch Dis Child 67: 340-344, 1992.

41 - BAKER SS; LIPTAK GS; COLETTI RB; CROFFIE JM; DILORENZO C; ECTOR W \& NURKO S. Constipation in infants and children: evaluation and treatment. J Pediatr Gastroenterol Nutr 29: 12-26, 1999.

42 - MOREIRA FL; COELHO CAR \& MAFFEI HVL. Constipação intestinal crônica em crianças atendidas no ambulatório de gastroenterologia infantil da Faculdade de Medicina de Botucatu-UNESP (1977- 1980). J Pediatr (Rio de Janeiro) 57: 62-65, 1984.

43 - GLEGHORN EE; HEYMAN MB \& RUDOLPH CD. No-enema therapy for idiopathic constipation and encopresis. Clin Pediatr 30: 669-672, 1991.

44 - FIBRAS alimentares. Rev Paul Pediatr 16: 169-170, 1998.

45 - MAFFEI HVL; MOREIRA FL; KISSIMOTO M; CHAVES SMF; EL FARO S \& ALEIXO AM. História clínica e alimentar de crianças atendidas em ambulatório de gastroenterologia pediátrica (GEP) com constipação intestinal crônica funcional (CICF) e suas complicações. J Pediatr (Rio de Janeiro) 70: 280-286, 1994.

46 - BARBIERI D. Constipação intestinal crônica inespecífica do lactente: um problema a ser explorado. J Pediatr (Rio de Janeiro) 78: 185-186, 2002.

47 - AGUIRRE ANC; VITOLO MR; PUCCINI RF \& MORAIS MB. Constipação em lactentes: influência do tipo de aleitamento e da ingestão de fibra alimentar. J Pediatr (Rio de Janeiro) 78: 202-208, 2002

48 - MAFFEI HVL \& JAEHN SM. Encoprese e escape fecal: conceitos e implicações terapêuticas. J Pediatr (Rio de Janeiro) 69: 155-158, 1993

49 - WALD A; CHANDRA R; GABEL S \& CHIPONIS DARLEEN. Evaluation of biofeedback in childhood encopresis. J Pediatr Gastroenterol Nutr 6: 554-558, 1987.

50 - WEBER J; DUCROTTE PH; TOUCHAIS JY; ROUSSIGNOL C \& DENIS, PH. Biofeedback training for constipation in adults and children. Dis Colon Rectum 30: 844-846, 1987.

51 - LOENING- BAUCKE VA. Modulation of abnormal defecation dynamics by biofeedback treatment in chronically constipated children with encopresis. J Pediatr 116: 214-222, 1990.

52 - DAVILA E; DE RODRIGUEZ GG; ADRIANZA A; PEREIRA Y; TORO J; GONZALES I \& ZÖMER H. Utilidad del biofeedback em niños com encopresis. Comunicacion preliminar. Gen 46: 297-301, 1992.

53 - BENNINGA MA; BÜLLER HÁ \& TAMINIAU JAJM. Biofeedback training in chronic constipation. Arch Dis Child 68:126129, 1993.

54 - LOENING- BAUCKE VA. Biofeedback treatment for chronic constipation and encopresis in childhood: long term outcome. Pediatrics 96: 105-110, 1995 
55 - CHIOTAKAKOU- FALIAKOU E; KAMM MA; ROY AJ; STORRIE JB \& TURNER IC. Biofeedback provides long term benefit for patients with intractable, slow and normal transit constipation. Gut 42: 517-521, 1998.

56 - GRIFFITHS DM \& MALONE PS. The malone antegrade continence enema. J Pediatr Surg 30: 68-71, 1995.

57 - RINTALA R.J \& LINDAHL H. Is normal bowel function possible after repair of intermediate and high anorectal malformations? J Pediatr Surg 30: 491-494, 1995.

58 - PENNINCKS F; LESTAR B \& KERREMANS R. Surgery for constipation: irrational things for desesperate people. Hepato-gastroenterology 37: 580-584, 1990.

59 - STABILE G; KAMM MA; PHILLIPS RKS; HAWLEY PR \& LENNARD-JONES JE. Partial colectomy and coloanal anastomosis for idiopathic megarectum and megacolon. Dis CoIon Rectum 35: 158-162, 1992.
60 - STEWARD J; KUMAR D; KEIGHLEY MRB. Results of anal or low rectal anastomosis and pouch construction for megarectum and megacolon. Br J Surg 81:1051-1053, 1994.

61 - KREBS C \& ACUÑA R. Transanal internal sphincter myomectomy: indications, operative procedure and results. Eur $\mathbf{J}$ Pediatr Surg 4: 151-167, 1994.

62 - TAITTZ LS; WALES JKH; URWIN OM \& MOLNAR D. Factors associated with outcome in management of defecation disorders. Arch Dis Child 61: 472-477, 1986.

Recebido para publicação em 07/07/2003

Aprovado para publicação em 24/11/2003 